\title{
Portable Accelerometer-based System for Aiding Elbow Extension in Post-Stroke Individuals
}

\author{
Valentina A. Bakhtina ${ }^{1}$, Pavel S. Marinushkin ${ }^{1}$, Alexey A. Levitskiy ${ }^{1}$, Professional Member, IEEE, \\ Alexandra A. Ilminskaya ${ }^{2}$, Mariia V. Abroskina ${ }^{2}$, Semyion V. Prokopenko ${ }^{2}$ \\ ${ }^{1}$ School of Engineering Physics and Radio Electronics, Siberian Federal University, Krasnoyarsk, Russia \\ ${ }^{2}$ The Department of Nervous Diseases with a Course of Medical Rehabilitation in Postgraduate Education, \\ Prof. V. F. Voino-Yasenetsky Krasnoyarsk State Medical University, Krasnoyarsk, Russia
}

\begin{abstract}
In this work, we design a wearable system providing control of elbow extension in hemiparetic individuals suffering from the post-stroke effects by stimulation of the triceps brachii muscle. Proposed system uses low-cost consumer grade MEMS accelerometer attached to the individual's forearm and measuring the tilt angle of the forearm relative to the gravity vector. Flexing the elbow above a predetermined threshold flexion angle triggers the triceps electrical stimulation. Among other things, the system is capable to transmit inertial data to external devices via Bluetooth. The operability of the system has been validated with healthy individuals and the results showed its potential as a tool for upper extremity rehabilitation after stroke.
\end{abstract}

Index Terms - Accelerometer; functional electrical stimulation (FES); microelectromechanical systems (MEMS); rehabilitation; wearable sensors.

\section{INTRODUCTION}

$\mathrm{S}^{\mathrm{T}}$ TROKE is among the major health problems of the adult population. Even with a favorable outcome, it is far from always possible to return to work and a former way of life. Many survivors are left with impairments and disabilities of different severity requiring long-lasting rehabilitation to improve. Typically, patients suffer from one-sided weakness, known as hemiparesis. Often sufferers develop pathological synergies, also known as Wernicke Mann posture characterized by the upper limb flexed and the lower limb extended and externally rotated.

The motor functions of the affected limbs can be improved by intensive exercise and augmented feedback. To address this problem, numerous devices have been developed to facilitate recovering. Wladimir T. Liberson et al. [1] were the first to apply short electrical pulses (functional electrical stimulation, FES) via skin-surface electrodes to activate weakened muscles of the foot during the swing phase of gait thus supporting foot drop patients. Proposed neuroprosthesis used artificial feedback based on force sensing resistor deployed into shoe to detect footfloor contact and to automatically trigger a stimulation. Later, the use of FES in the lower extremities of the body has been investigated intensively and successfully adopted for balancing, standing and walking correction [2, 3], helping to improve the lifestyle of neurologically impaired individuals.

Along with other treatments targeting motor and sensory functions, improving the function of the upper limbs is crucial for the patient's rehabilitation since hands and arms are involved in the majority of the basic activities of daily living. In this respect, FES application to upper motor rehabilitation has the following therapeutic goals $[4,5]$ : facilitating recovery process, maintaining or enhancing the trophic state of the muscles, modifying altered patterns of automatic and volitional functional movement, reducing spasticity, strengthening weak muscles, increasing range of motion, improving motor control.

Among the early works that demonstrated the possibility of using electrotherapy for restoring or improving grasping function was transcutaneous hand neuroprosthesis system proposed by Rebersek and Vodovnik [6]. This system used three stimulation channels to generate the grasping functions by stimulating the finger and thumb flexors and extensors. It is worth mentioning that this device allowed controlling the stimulation intensity through feedback from the electromyography sensor, variable potentiometer and pressure transducer. This work was quickly followed by Merletti et al. [7] who described a similar system with cutaneous electrodes implementing a two channel functional electrical stimulation in order to improve the hand opening and elbow extension in hemiplegic patients.

Many more stimulation systems for functional support of the upper extremities have been developed since [8-17]. Summarizing, all existing upper-limb neuroprostheses consist of a stimulator activating the muscles in the upper limb, an input transducer, and a control unit [10].

More recent studies investigate the implementation of new control methods and advanced MEMS sensors technologies to further facilitate FES clinical implementation and enhance its efficiency. In particular inertial MEMS sensors, such as accelerometers and gyroscopes were introduced for providing the input data needed for FES control [17].

While many devices have been developed to facilitate hand rehabilitation, only few address both posture and function correction in proximal arm while also enabling work in motion. Assistive systems existing at the present time work mainly with distal arms having limited use for 
persons with severe paresis, moderate / severe cognitive impairment, depression. Therefore, additional research and development is required in this field to improve upper extremity rehabilitation technology.

\section{PROBLEM FORMULATION}

One of the FES main focuses for upper limb is elbow extension $[18,19]$ which assumes bringing the forearm of the impaired limb back to anatomical position. Point is that the elbow position control is often impaired in patients with hemiparesis due to deficit of the antagonistic muscles regulation. While the elbow flexors are spastic, the elbow extensors are weakened.

To achieve elbow extension a FES-based assistive device should provide measuring the elbow position and activating the contraction of the triceps brachii muscle when the elbow angle is above than the specified threshold value. This can be done by attaching to an individual's forearm a tilt sensor triggering the stimulation according to the measurement, as shown in Fig. 1. Along with the tilt sensor, a stimulator fitted with electrodes is attached to the forearm. Electrical stimulation of the triceps muscle induces extension force which exerts elbow extension. Control is achieved through the relationship between the position of the forearm and the intensity of stimulation.

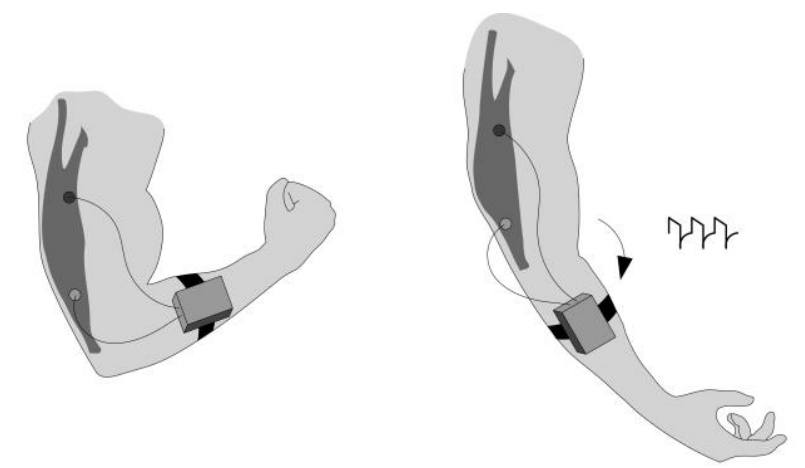

Fig. 1. The proposed wearable rehabilitation system. The rehabilitation device attached by the strap to an individual's forearm uses a tilt sensor to trigger elbow extension when the elbow angle is above a predetermined threshold.

\section{DESIGN CONSIDERATIONS}

\section{A. Measurement Unit}

Implementation of the described rehabilitation system requires an elbow position transducer to measure the elbow angle and generate a command signal for elbow extension. Due to their small size and low power consumption, MEMS-based inertial sensors are well suited for this role.

The simplest approach to elbow angle tracking assumes calculating the tilt of the MEMS accelerometer from the vertical defined by the gravity vector direction (Fig. 2). The advantage of this method is its insensitivity to alignment errors of the measurement unit relative to the forearm. Therefore, no pre-calibration of the measuring system is required after it has been donned. However, due to the lack of a reference orientation, an obvious disadvantage is the inability to distinguish between different anatomical rotations and to ensure the absolute value of the angle. Nevertheless, for exercises implying a vertical position of the patient this method is quite acceptable.

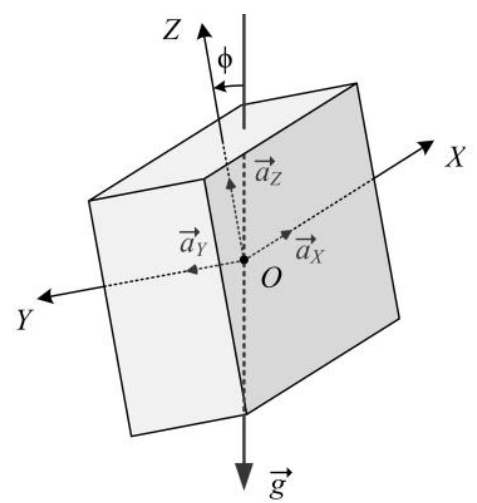

Fig. 2. Coordinate system of the accelerometer. Three mutually perpendicular axes $(X, Y$ and $Z$ ) intersect at the point $O$. $Z$-axis is orthogonal to the horizon.

Assuming slow motions, MEMS accelerometer provides only the $X, Y$ and $Z$ components of the gravity vector: $a_{\mathrm{X}}$, $a_{\mathrm{Y}}, a_{\mathrm{Z}}$. Then the tilt angle between the gravity vector and the $Z$ axis of the accelerometer (and forearm it is attached to) can be calculated from the accelerometer's outputs using the following expression [20]:

$$
\phi=\arctan \left(\frac{\sqrt{a_{X}^{2}+a_{Y}^{2}}}{a_{Z}}\right) .
$$

The task of converting the raw acceleration data into the tilt angle value is performed by the microcontroller unit (MCU).

\section{B. Configuration of the Stimulation Unit}

Electrical stimulation unit consists of DC-DC boost converter and full bridge output stage. Step-up DC-DC voltage converter boosts the battery's $9 \mathrm{~V}$ supply by 3 times at the output stages, which is required for muscles excitation. Command pulses from the controller drive output stage. To avoid MCU damage the outputs of the measurement unit are galvanically isolated from the stimulation unit using optocouplers.

To meet the charge balance requirement, a symmetrical two-phase waveform was chosen as the pulse shape for stimulation.

\section{PRACTICAL IMPLEMENTATION}

Prototype of the proposed FES system consists of a control/measurement and electrical stimulation units, surface conductive electrodes and cabling (Fig. 3). The overall system operates by a single $9 \mathrm{~V}$ battery.

Measurement unit is mounted in a custom designed case approximately $7.2 \times 5.6 \times 2.5 \mathrm{~cm}$. The control/measurement unit is presented by the board 9DoF Razor IMU M0 
equipped with MPU9250 multi-chip module from InvenSense Inc. It collects inertial data with sampling rate $100 \mathrm{~Hz}$. The onboard algorithm carries out preliminary calibration and processes inertial measurements data stream. Measured accelerations and calculated tilt angle can be transferred via UART to Bluetooth module and then wirelessly to a computer. Thus monitoring of the rehabilitation progress is possible after each training session.

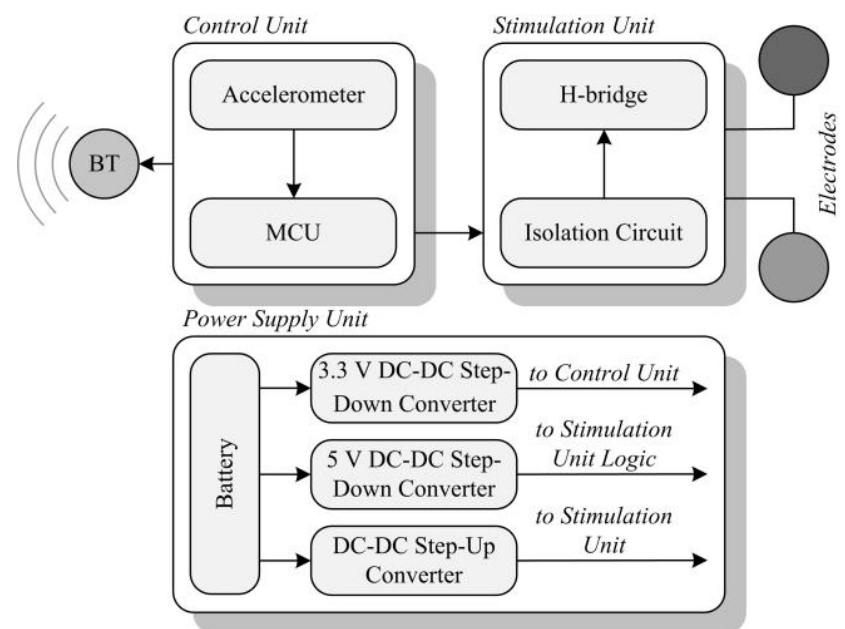

Fig. 3. FES system includes control/measurement and stimulation units, a power supply, accelerometer and a means for providing Bluetooth wireless communication. Based on signals received from accelerometer, MCU determines the tilt angle of a user's forearm (i.e., the angle between the user's forearm and a gravity vector), and provide electrical signals to electrodes at appropriate times.

Testing of the controller algorithm performance was carried out visually on healthy subjects. For this purpose measurement unit was attached to the forearm of the subject's affected arm using adjustable strap so that the Zaxis of the measurement unit was approximately aligned with the longitudinal axis of the forearm. Raw acceleration data and calculated value of the tilt were simultaneously sent to the computer via Bluetooth interface. Fig. 4 illustrates the changes in tilt angle that occur when flexing/extending the elbow.

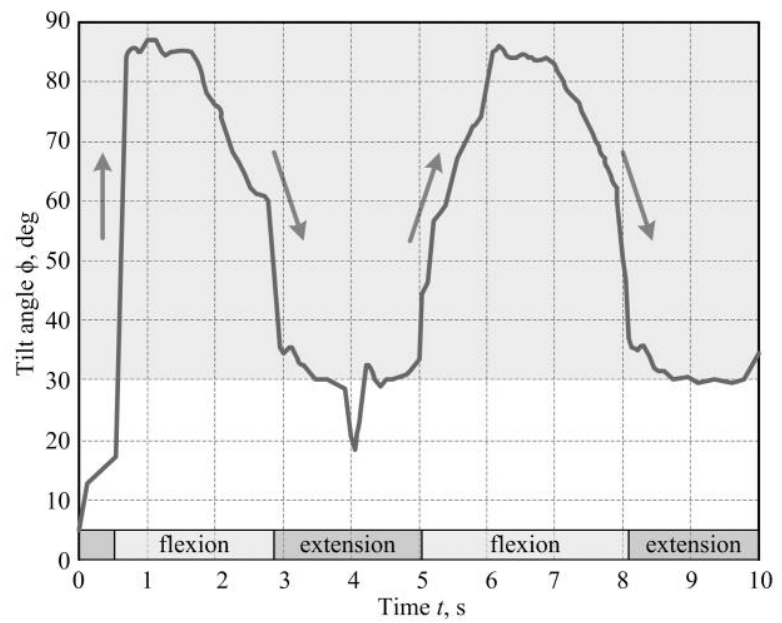

Fig. 4. Measurement unit output example. The threshold angle is set to $30^{\circ}$
Fig. 5 depicts the prototype of the stimulation unit. To switch the polarity of a stimulus pulses we used a L298N $\mathrm{H}$-bridge built with bipolar junction transistors. MC34063 switching regulator serves for DC-DC conversion. The resulting circuit produces biphasic stimulation pulses at predefined width and frequency can be operated in a single-channel configuration with a $25 \mathrm{~mA}$ stimulation current.

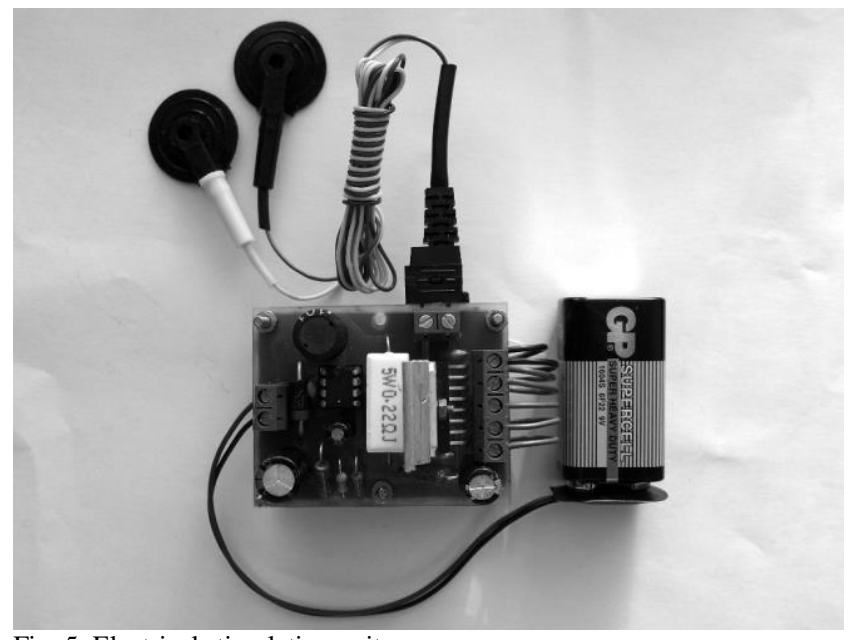

Fig. 5. Electrical stimulation unit.

To check the output pulses sequence generated by the stimulation unit, the output signals were applied to a resistive load, after which the voltage signals on the resistor were measured with an oscilloscope (Fig. 6). The output delivers approximately $55 \mathrm{~V}$ peak-peak for a $1 \mathrm{k} \Omega$ load.

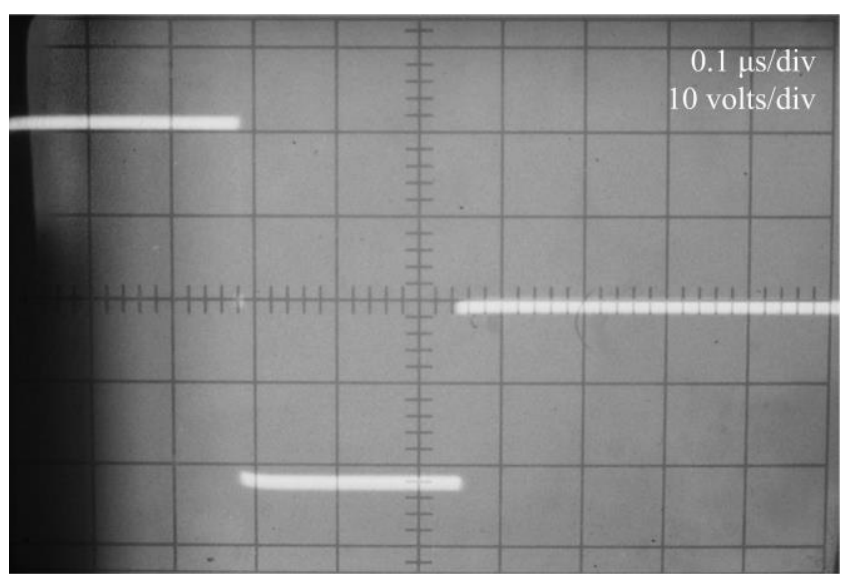

Fig. 6. Stimulation unit output example. The stimulation frequency is set to $50 \mathrm{~Hz}$, and the pulse width is $250 \mu \mathrm{s}$.

\section{CONCLUSION}

Rehabilitation system has been developed to provide the post-stroke individuals with control of elbow position. The experiments performed indicate the feasibility of the proposed system: it allows to perform rehabilitation procedures that require only minimal participation by the therapist, and is potentially suitable for home use. More 
detailed clinical tests providing a quantitative measure of the system effectiveness and functionality are under development. Further work is planned in determining the optimal electrical stimulation parameters for developing elbow extension movements during rehabilitation procedures. Correspondingly, the implemented technical solutions have to be thoroughly verified for insurance of patient safety. Finally, robust algorithmic schemes should be developed in order to enable the system operation under the limitations of the real clinical case scenarios.

\section{ACKNOWLEDGMENT}

The authors thank Dmitrii E. Goncharov for helpful technical discussion.

\section{REFERENCES}

[1] W. T. Liberson, H. J. Holmquest, D. Scot, M. Dow, Functional electrotherapy: Stimulation of the peroneal nerve synchronized with the swing phase of the gait of hemiplegic patients". Archives of Physical Medicine and Rehabilitation. 42, pp. 101-105, 1961. PMID: 13761879

[2] G. M.Lyons, T. Sinkjaer, J. H. Burridge, D. J. Wilcox, "A review of portable FES-based neural orthoses for the correction of drop foot." IEEE Transactions on Neural Systems and Rehabilitation Engineering, vol. 10, no. 4, pp. 260-279, 2002 DOI: $10.1109 /$ TNSRE. 2002.806832

[3] P. L. Melo, M. T. Silva, J. M. Martins, D. J. Newman, "Technica developments of functional electrical stimulation to correct drop foot: Sensing, actuation and control strategies," Clinical Biomechanics, Volume 30, Issue 2, pp. 101-113, 2015 DOI: 10.1016/j.clinbiomech.2014.11.007.

[4] M. M. Dimitrijevic, M. R. Dimitrijevic, "Clinical Elements for the Neuromuscular Stimulation and Functional Electrical Stimulation Protocols in the Practice of Neurorehabilitation". Artificial Organs vol. 26, No. 3, pp. 256-259, 2002. DOI: 10.1046/j.15251594.2002.06946.x

[5] https://www.physio-pedia.com/Electrical_Stimulation_Its_role_in_upper_limb_recovery_post-stroke

[6] S. Rebersek, L. Vodovnik, "Proportionally controlled functional electrical stimulation of hand". Archives of Physical Medicine and Rehabilitation, vol. 54, pp. 168-172, 1973. PMID: 4269119

[7] R. Merletti, R. Acimovic, S. Grobelnik, G. Cvilak, "Electrophysiological orthosis for the upper extremity in hemiplegia: feasibility study." Archives of Physical Medicine and Rehabilitation, vol. 56, No. 12, pp. 507-513, 1975. PMID: 1081869

[8] R. H. Nathan, "Device for generating hand function," U.S. Patent $5330516,1994$.

[9] A. Prochazka, M. Wieler, Z. Kenwell, M. Gauthier, "Garment for applying controlled electrical stimulation to restore motor function," U.S. Patent 5562 707, 1996.

[10] Alon G, Dar A, Katz-Behiri D, Wingarden H, Nathan R. Efficacy of a hybrid upper limb neuromuscular electrical stimulation system in lessening selected impairments and dysfunctions consequent to cerebral damage. J Neurol Rehabil, 2, pp. 73-80, 1998.

[11]M. Milenkovic, E. Jovanov, J. Chapman, D. Raskovic and J. Price, "An accelerometer-based physical rehabilitation system," Proceedings of the Thirty-Fourth Southeastern Symposium on System Theory (Cat. No.02EX540), Huntsville, AL, USA, 2002, pp. 57-60 DOI: $10.1109 /$ SSST.2002.1027005

[12]G. Alon, K. McBride, H. Ring, Improving selected hand functions using a noninvasive neuroprosthesis in persons with chronic stroke. J Stroke Cerebrovasc Dis 2002;11:99-106.

[13]L. R. Sheffler, J. Chae, "Neuromuscular electrical stimulation in neurorehabilitation. Muscle Nerve,” 35, pp. 562-590, 2007. DOI: $10.1002 /$ mus. 20758
[14]R. D. Willmann et al., "Home Stroke Rehabilitation for the Upper Limbs," 29th Annual International Conference of the IEEE Engineering in Medicine and Biology Society, pp. 4015-4018, 2007. DOI: 10.1109/IEMBS.2007.4353214

[15]C. Klauer, T. Schauer, J. Raisch, "High Performance Motion Control by Neuro-Muscular Electrical Stimulation applied to the UpperLimb," Proceedings of 15th Annual International FES Society Conference and 10th Vienna Int. Workshop on FES, pp. 318-320, 2010

[16]P. Müller et al., "Alignment-free, self-calibrating elbow angles measurement using inertial sensors," Proceedings of the IEEE-EMBS International Conference on Biomedical and Health Informatics (BHI), pp. 583-586, 2016

[17]S. I. Lee et al., "Enabling Stroke Rehabilitation in Home and Community Settings: A Wearable Sensor-Based Approach for UpperLimb Motor Training," IEEE Journal of Translational Engineering in Health and Medicine, vol. 6, pp. 1-11, 2018, Art no. 2100411. DOI: $10.1109 /$ JTEHM.2018.2829208

[18]P. E. Crago et al., An elbow extension neuroprosthesis for individuals with tetraplegia, IEEE transactions on rehabilitation engineering, 1998, 6(1), pp. 1-6

[19]W.D. Memberg, P.E. Crago, M.W. Keith, "Restoration of elbow extension via functional electrical stimulation in individuals with tetraplegia," J Rehabil Res Develop 2003; 40: 477-486.

[20]C. J. Fisher, "Using an Accelerometer for Inclination Sensing." AN1057 Application note, Analog Devices, 2010.

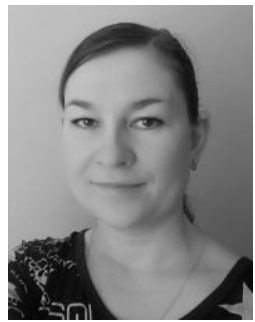

Valentina A. Bakhtina received B.S. and M.S. degrees in electrical engineering from the Krasnoyarsk State Technical University, Krasnoyarsk, and Siberian Federal University, Krasnoyarsk, in 2006 and 2008, respectively. Currently she is pursuing a Candidate of Sciences degree at the School of Engineering Physics and Radio Electronics of Siberian Federal University.

Her research interests include microelectronic technologies.

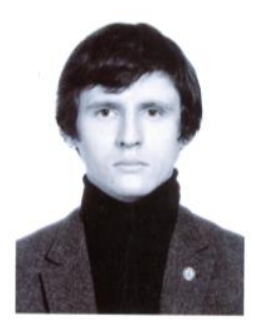

Pavel S. Marinushkin received B.S. and M.S. degrees in electrical engineering from the Krasnoyarsk State Technical University, Krasnoyarsk, and Siberian Federal University, Krasnoyarsk, in 2006 and 2008, respectively. During the 2011 Spring Semester he was a PG Visiting Internship Student at Hong Kong University of Science and Technology. He received the Candidate of Engineering Sciences degree in specialty "Devices and methods for control of environment, substances, materials and products" from Omsk State Technical University, Omsk, in 2015.

In 2016/2017 Fall Semester he was a Guest Scientist with the Ilmenau University of Technology. Since 2016, he has been an Assistant Professor with the Department of Instrumentation and Nanoelectronics, Siberian Federal University, Krasnoyarsk. His research interests include microelectromechanical systems (MEMS) and applications.

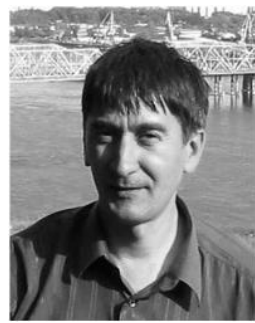

Alexey A. Levitskiy (M'09) received Candidate of Engineering Sciences degree in Radiophysics from Leningrad Electrotechnical University in 1998. Currently he is a Head of the Department of Instrumentation and Nanoelectronics at the School of Engineering Physics and Radio Electronics of Siberian Federal University.

His current research interests include MEMS design and applications. 


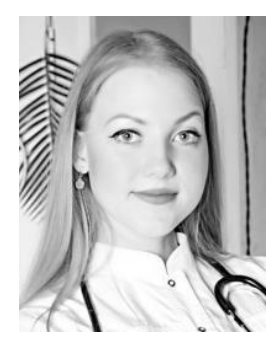

Alexandra A. Ilminskaya is the resident of the Department of Nervous Diseases Krasnoyarsk State Medical University.

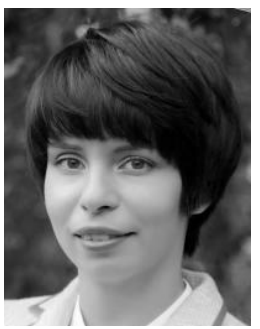

Mariia V. Abroskina received academic degree of Candidate of Medical Sciences by specialty of Nervous diseases from Krasnoyarsk State Medical University, in 2014.

Currently, she is the Associate Professor of the Department of Nervous Diseases Krasnoyarsk State Medical University and the Neurologist in Federal State Budgetary Institution Siberian Federal Research and Clinical Center of the Federal Medical and Biological Agency of Russia.

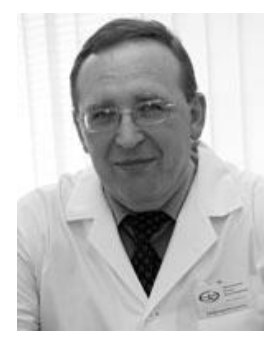

Russia.
Semyion V. Prokopenko received academic degree of Doctor of Medical Sciences by specialty of Nervous diseases from Krasnoyarsk State Medical University, in 2000.

Currently, he is the Head of the Department of Nervous Diseases Krasnoyarsk State Medical University and the chief scientific consultant in Federal State Budgetary Institution Siberian Federal Research and Clinical Center of the Federal Medical and Biological Agency of 